www.nature.com/clinicalpractice/onc

and Ovarian Cancer Screening Trial. Serum vitamin $\mathrm{D}$ concentrations were measured in blood samples taken at enrollment; all prostate cancer cases included in this analysis were diagnosed 1-8 years after the blood draw.

The researchers found no statistically significant difference in the risk of prostate cancer with increasing vitamin $\mathrm{D}$ concentration. They did see some evidence of an increased risk of aggressive disease (stage III or IV tumors or a Gleason sum $\geq 7$ ) associated with high vitamin $D$ concentrations, but this trend was not statistically significant and the association did not show a linear dose-response relationship.

These results do not support the hypothesis that increased serum vitamin $D$ levels decrease the risk of prostate cancer. Further studies are required to confirm these results and clarify the effects of vitamin $D$ on prostate cancer.

Original article Ahn J et al. (2008) Serum vitamin D concentration and prostate cancer risk: a nested case-control study. J Natl Cancer Inst 100: 796-804

\section{Liver resection with salvage transplantation for hepatocellular carcinoma}

Many patients with hepatocellular carcinoma (HCC) experience disease progression while on the transplant waiting list and are no longer eligible for liver transplantation (LT) when an organ becomes available. Liver resection (LR), with salvage $L T$ for patients who experience
HCC recurrence, has been shown to produce similar survival outcomes to first-line LT and might be an appropriate treatment strategy for some patients with HCC.

Del Gaudio et al. compared the efficacy and safety of $L R$ and salvage $L T$ versus $L T$ in patients with cirrhosis and $\mathrm{HCC}$ who were suitable candidates for LT. First-line LT was performed in 147 patients, whereas 80 patients with preserved hepatic function underwent potentially curative LR. HCC recurred in 39 of the patients in the LR group and salvage LT was performed in 16 (10 for tumor recurrence and 6 for hepatic decompensation), whereas the remainder were considered untreatable by LT.

No significant difference in 5-year overall survival was found between patients who underwent LR (with or without salvage LT) and those who underwent LT (66\% vs $73 \%$ ), although 5 -year disease-free survival was lower in the LR group (41\% vs $71 \%$; $P=0.001)$. Operative mortality, tumor recurrence, 5 year overall and disease-free survival were all similar in LR patients who underwent salvage LT and those who underwent first-line LT

The authors conclude that LR in patients with cirrhosis and HCC who are suitable candidates for transplant, with salvage LT in cases of recurrence or liver decompensation, is a safe and effective alternative to LT.

Original article Del Gaudio M et al. (2008) Liver transplantation for recurrent hepatocellular carcinoma on cirrhosis after liver resection: University of Bologna experience. Am J Transplant 8: 1177-1185 